\title{
High surface area vanadium phosphate catalysts for n-butane oxidation.
}

\begin{abstract}
Vanadium phosphorus oxide (VPO) was prepared using the precipitation procedure and tested for potential use in the partial oxidation reaction of n-butane to maleic anhydride. In particular, the effect of reducing agents such as the isobutanol, 1-butanol, and glycol, subsequent water treatment, and microwave heating were investigated in detail. The optimum synthesis conditions were identified with respect to catalyst activity for the oxidation of nbutane. The activity and selectivity of VPO prepared catalysts have been evaluated in a fixed bed microreactor and in situ gas chromatography (GC) was used to evaluate the system efficiency and analyze the product effluent stream. The different catalysts exhibited a range of activities and selectivities under the same reaction conditions. The range in catalyst performance may be attributed to the crystal size as well as particle size of catalyst. The results were interpreted in terms of surface area and catalyst nanostructure, and it has been generally concluded that the catalyst surface area is enhanced by the employment of glycol as the reducing agent, followed refluxing by distilled water and drying by microwave irradiation. The catalyst produced using this method is the most active and selective catalyst for partial oxidation of n-butane to maleic anhydride. The catalyst lifetime was tested under the optimum reaction conditions, and the catalyst was found to be highly stable for more than 70 h. The characterization of both precursors and calcined catalysts was carried out using X-ray diffraction (XRD), inductively coupled plasma-atomic emission spectrometer (ICP-AES), Brunauer-Emmer-Teller (BET) surface area measurement, temperature programmed reduction (H2-TPR), and scanning electron microscopy (SEM).
\end{abstract}

Keyword: Vanadium phosphorus oxide (VPO); n-butane; Industrial organic chemicals. 